\title{
A Hybrid Waveguide Sensor for Highly Sensitive Biosensing
}

\author{
M. Z. Alam, F. Bahrami, J. S. Aitchison and M. Mojahedi \\ Department of Electrical and Computer Engineering, \\ University of Toronto, Toronto, Ontario, Canada, MSS $3 G 4$ \\ malam@waves.utoronto.ca
}

\begin{abstract}
We propose a highly sensitive biosensor consisting of a metal-plane separated from a silicon layer by a nano-fludic channel. The sensor performance is estimated and a method to distinguish bulk and surface sensitivity is proposed.

OCIS codes: (280.1415) Biological sensing and sensors; (310.6628) Subwavelength structures, nanostructures; (130.3120) Integrated optics.
\end{abstract}

\section{Introduction}

Surface plasmons (SP) are currently one of the most widely used techniques for affinity biosensing. Because of the high field confinement of SP, these sensors are extremely sensitive and the presence of gold as a part of the guiding structure makes them suitable for functionalization with a wide range of antigen and antibodies- a great advantage for biosensing. Commonly used SP based sensors depend on only one measurement (e.g. shift in reflection minimum in an attenuated total reflection configuration) and cannot differentiate between the change of adlayer thickness and change of bulk sample properties, for example variation of index of water with temperature. Though a reference channel can be used to alleviate this problem, even a small degree of mismatch between the reference and sensing arm may compromise the effectiveness of such a scheme [1]. As a result very precise control of temperature is necessary for the proper operation of a SP sensor. Dielectric waveguide sensors capable of measuring the change of both TM and TE mode properties do not suffer from this limitation [2] but the difficulty of functionalization of the dielectric surface makes them less useful for biological applications [3]. Here we propose a new biosensing scheme that uses the recently proposed hybrid guide [4-6] to combine the benefits of SP and dielectric waveguide sensors.

\section{Analysis and Results}

Figure 1(a) shows the hybrid waveguide that acts as the guiding structure for the proposed biosensing scheme. It consists of a thin silicon film of thickness $d$ separated from a gold surface by a nano-fluidic channel of thickness $h$. Figures 1(b) and (c) show the guided power density profiles calculated using finite element code Comsol Multiphysics for the TE and TM modes. The presence of both TE and TM modes offers the possibility of two independent measurements. The presence of the gold surface also makes the sensor very suitable for biological applications. Here we present only the sensitivity analysis for a one dimensional hybrid guide. Details of specific implementations (prism and grating coupling, Mach-Zehnder interferometer) will be provided in our presentation.

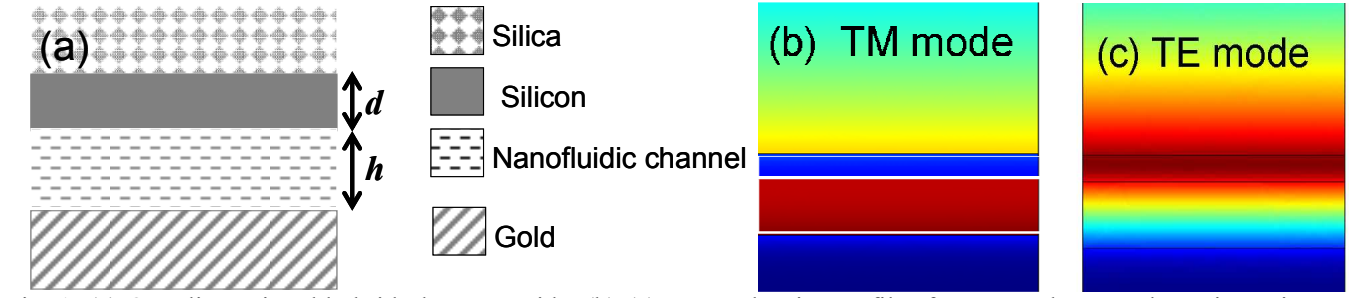

Fig. 1: (a) One dimensional hybrid plasmon guide. (b)-(c) Power density profiles for TM and TE modes. Dimensions are $h=100 \mathrm{~nm}, d=50 \mathrm{~nm}$. Wavelength of operation is $1.17 \mu \mathrm{m}$.

Figure 2 shows the bulk sensitivity $\left(\partial n_{e f f} / \partial \mathrm{n}_{\mathrm{c}}\right)$ and surface sensitivity $\left(\partial n_{e f f} / \partial a\right)$ for both the TM and TE modes. Here $n_{\text {eff }}, a$ and $n_{c}$ are the effective mode index, thickness of adlayer attached to the gold surface and the index of the fluid in the nano-fluidic channel respectively. For a $100 \mathrm{~nm}$ channel thickness surface and bulk sensitivities of the TM mode are $7.8 \times 10^{-4}\left(\mathrm{~nm}^{-1}\right)$ and 0.482 , respectively. The surface sensitivity is more than 2.5 times that of single interface SP [7] at the same wavelength but the bulk sensitivity is half that of single interface SP. High surface sensitivity and low bulk sensitivity makes the sensor suitable as an affinity sensors since it suffers less from the undesirable effect of fluctuation of bulk sample, while remaining highly sensitive to thickness variation of adlayer. Both bulk and surface sensitivity of the TE mode are an order of magnitude smaller than that of TM mode; however, an effective index change as small as $5 \times 10^{-6}$ can readily be detected by a suitable detection scheme [3]. In other words, the change of effective index for TE mode is also detectable, even for very small changes in adlayer 
thickness. We would like to add that fluid flow through a channel much narrower than $100 \mathrm{~nm}$ has been experimentally demonstrated before [8] and hence the proposed structure has applications in bio-molecular sensing.


Fig. 2. Variations of sensitivity with channel thickness (a) Surface sensitivity of the TE and TM modes. (b) Bulk sensitivity of the TM and TE modes. Silicon thickness is $40 \mathrm{~nm}$ and wavelength of operation is $1.17 \mu \mathrm{m}$.

Since the plasmonic hybrid guide can support both TM and TE modes, the contributions from bulk and surface sensitivities can be separated and the determination of the adlayer thickness can be further improved. Following the procedure described in [2], the variation of bulk index of the fluid $\left(\Delta n_{c}\right)$ and the variation of thickness of adlayer $(\Delta a)$ can be expressed in terms of the change of effective index for the fundamental TE mode $\Delta N\left(T E_{0}\right)$ and for the fundamental TM mode $\Delta N\left(T M_{0}\right)$ as

$$
\begin{aligned}
& \Delta n_{c}=\frac{1}{\frac{\partial n_{\text {eff }}}{\partial n_{c}}\left(T E_{0}\right) \frac{\partial n_{\text {eff }}}{\partial a}\left(T M_{0}\right)-\frac{\partial n_{\text {eff }}}{\partial a}\left(T E_{0}\right) \frac{\partial n_{\text {eff }}}{\partial n_{c}}\left(T M_{0}\right)}\left[\frac{\partial n_{\text {eff }}}{\partial a}\left(T M_{0}\right) \Delta N\left(T E_{0}\right)-\frac{\partial n_{\text {eff }}}{\partial a}\left(T E_{0}\right) \Delta N\left(T M_{0}\right)\right] \\
& \Delta a=\frac{1}{\frac{\partial n_{\text {eff }}}{\partial n_{c}}\left(T E_{0}\right) \frac{\partial n_{e f f}}{\partial a}\left(T M_{0}\right)-\frac{\partial n_{e f f}}{\partial a}\left(T E_{0}\right) \frac{\partial n_{e f f}}{\partial n_{c}}\left(T M_{0}\right)}\left[-\frac{\partial n_{e f f}}{\partial n_{c}}\left(T M_{0}\right) \Delta N\left(T E_{0}\right)+\frac{\partial n_{e f f}}{\partial n_{c}}\left(T E_{0}\right) \Delta N\left(T M_{0}\right)\right]
\end{aligned}
$$

For the channel thickness of $100 \mathrm{~nm}$ and a $40 \mathrm{~nm}$ thick silicon film, the relations becomes

$$
\begin{gathered}
\Delta n_{c}=9.25 \times \Delta N\left(T E_{0}\right)-0.2864 \times \Delta N\left(T M_{0}\right) \\
\Delta a=-5738 \times \Delta N\left(T E_{0}\right)+1464 \times \Delta N\left(T M_{0}\right)
\end{gathered}
$$

Once $\Delta N\left(T E_{0}\right)$ and $\Delta N\left(T M_{0}\right)$ are known from measurements, $\Delta n_{c}$ and $\Delta a$ can be determined from equations (3) and (4).

\section{Conclusion}

We have proposed a new sensing scheme that is highly sensitive and offers the possibility of extracting information about both adlayer and bulk properties from measurements of both TE and TM modes. It is also suitable for silicon on insulator based integrated optics fabrication technology. Though not explained here, by choosing a thicker silicon film, it is possible to guide higher order modes as well and measuring the changes of properties of those modes can provide additional information, for example adlayer refractive index - properties which need to be known a priori for SP based biosensing scheme.

\section{References}

[1] A. N. Naimushin, S. D. Soelberg, D. W. Batrholmew and J. L. Elkind, C. E. Furlong, "A portable surface plasmon resonance (SPR) sensor system with temperature regulation," Sens. Act. B 96, 253-260 (2003).

[2] K. Tiefenthaler, W. Kukosz, "Sensitivity of grating couplers as integrated-optical chemical sensors," JOSA.B 6, $209-220$ (1989).

[3] P. M. Nellen, K. Tiefenthaler and W.Lukosz,"Integrated optical input grating couplers as biochemical sensors," Sens. Act. 15, 285-295(1988).

[4] M Z. Alam, J. Meier, J S. Aitchison and M Mojahedi, "Super mode propagation in low index medium," CLEO/QELS (2007).

[5] M. Z. Alam, J. Meier, J. S. Aitchison and M. Mojahedi, "Propagation characteristics of hybrid modes supported by metal-low-high index waveguides and bends," Opt. Expr. 18, 12971-12979 (2010).

[6] R. F. Oulton, V. J. Sorger, D. A. Genov, D. F. P. Pile, X. Zhang, “A hybrid plasmonic waveguide for subwavelength confinement and long range propagation," Nature Photonics 2, 496-500 (2008).

[7] P. Berini, "Bulk and surface sensitivities of surface plasmon waveguides," New J. of Phys. 10, 105010 (2008).

[8] C.Wu, Z. Jin, H.Wang, H. Ma, Y.Wang, "Design and fabrication of a nanofluidic channel by selective thermal oxidation and etching back of silicon dioxide made on a silicon substrate", J. Micromech. Microeng. 17, 2393-2397 (2007). 